\title{
The effect of dietary asparagine supplementation on energy metabolism in liver of weaning pigs when challenged with lipopolysaccharide
}

\author{
Ping Kang ${ }^{1}$, Yulan Liu ${ }^{1, *}$, Huiling Zhu' ${ }^{1}$ Jing Zhang ${ }^{1}$, Haifeng Shi ${ }^{1}$, Shuang Li ${ }^{1}$, Dinan Pi ${ }^{1}$, Weibo Leng ${ }^{1}$, \\ Xiuying Wang', Huanting $\mathrm{Wu}^{1}$, and Yongqing Hou ${ }^{1}$
}

\author{
* Corresponding Author: Yulan Liu \\ Tel: +86-27-83956175, Fax: +86-27-83956175, \\ E-mail: yulanflower@126.com \\ ${ }^{1}$ Hubei Collaborative Innovation Center for Animal \\ Nutrition and Feed Safety, Hubei Key Laboratory \\ of Animal Nutrition and Feed Science, Wuhan \\ Polytechnic University, Wuhan 430023, China \\ ORCID \\ Ping Kang \\ https://orcid.org/0000-0003-1665-8862 \\ Yulan Liu \\ https://orcid.org/0000-0003-3320-2381
}

Submitted Jun 3, 2017; Revised Sept 8, 2017; Accepted Oct 22, 2017
Objective: This experiment was conducted to investigate whether asparagine (Asn) could improve liver energy status in weaning pigs when challenged with lipopolysaccharide. Methods: Forty-eight weaned pigs (Duroc $\times$ Large White $\times$ Landrace, $8.12 \pm 0.56 \mathrm{~kg}$ ) were assigned to four treatments: i) CTRL, piglets received a control diet and injected with sterile $0.9 \% \mathrm{NaCl}$ solution; ii) lipopolysaccharide challenged control (LPSCC), piglets received the same control diet and injected with Escherichia coli LPS; iii) lipopolysaccharide (LPS) $+0.5 \%$ Asn, piglets received a $0.5 \%$ Asn diet and injected with LPS; and iv) LPS+1.0\% Asn, piglets received a 1.0\% Asn diet and injected with LPS. All piglets were fed the experimental diets for $19 \mathrm{~d}$. On d 20, the pigs were injected intraperitoneally with Escherichia coli LPS at 100 $\mu \mathrm{g} / \mathrm{kg}$ body weights or the same volume of $0.9 \% \mathrm{NaCl}$ solution based on the assigned treatments. Then the pigs were slaughtered at $4 \mathrm{~h}$ and $24 \mathrm{~h}$ after LPS or saline injection, and the liver samples were collected.

Results: At $24 \mathrm{~h}$ after LPS challenge, dietary supplementation with $0.5 \%$ Asn increased ATP concentration (quadratic, $\mathrm{p}<0.05$ ), and had a tendency to increase adenylate energy charges and reduce AMP/ATP ratio (quadratic, $p<0.1$ ) in liver. In addition, Asn increased the liver mRNA expression of pyruvate kinase, pyruvate dehydrogenase, citrate synthase, and isocitrate dehydrogenase $\beta$ (linear, $\mathrm{p}<0.05$; quadratic, $\mathrm{p}<0.05$ ), and had a tendency to increase the mRNA expression of hexokinase 2 (linear, $\mathrm{p}<0.1$ ). Moreover, Asn increased liver phosphorylated AMP-activated protein kinase (pAMPK)/total AMP-activated protein kinase (tAMPK) ratio (linear, $\mathrm{p}<0.05$; quadratic, $\mathrm{p}<0.05$ ). However, at $4 \mathrm{~h}$ after LPS challenge, Asn supplementation had no effect on these parameters.

Conclusion: The present study indicated that Asn could improve the energy metabolism in injured liver at the late stage of LPS challenge.

Keywords: Asparagine; Energy Metabolism; Lipopolysaccharide; Piglets

\section{INTRODUCTION}

L-asparagine (Asn) is non-essential amino acid. However, previous studies have demonstrated that weaning rats required Asn over a short period [1], and Asn could stimulate growth and protein synthesis of weaning rats. In addition, Asn can stimulate enterocyte proliferation [2,3] and improve jejunal enterocyte ornithine decarboxylase (ODC) activity $[3,4]$. Asparagine can be synthesized from aspartate and glutamine (or ammonia) by asparagine synthetase in mammals $[5,6]$. However, in liver, the rate of Asn synthesis is much less because of the lower Asn synthetase activity [7].

Asn has similar structure to aspartate, and it can be deaminated to form aspartate in an ATP-dependent amidation reaction [8]. Liver plays a pivotal role in the metabolism of the 
whole-body energy [9], such as stimulating fatty acid oxidation and glucose generation. LPS challenge could induce liver injury [10], which could cause insufficient energy supply in the liver [11]. Xu et al [12] reported that energy was necessary for a successful stress response. Our previous study has shown that dietary supplementation of aspartate improved energy status in lipopolysaccharide (LPS)-injured liver of weaning pigs [13]. Accordingly, we hypothesized that Asn could influence the energy metabolism in the liver in LPS-challenge weaning pigs. In this study, we measured the hepatic adenylate purine concentrations and the mRNA expression of key enzymes involved in glycolysis, tricarboxylic acid (TCA) cycle and fatty acid oxidation as well as AMP-activated protein kinase (AMPK) signaling pathway to examine the influence of Asn on energy metabolism in the liver in LPS-challenged weaning pigs.

\section{MATERIALS AND METHODS}

\section{Animals}

The Animal Care and Use Committee of Wuhan Polytechnic University approved the animal use protocol for this research. This experiment was conducted in the Hubei Key Laboratory of Animal Nutrition and Feed Science (Wuhan Polytechnic University, Wuhan, China). In this study, a total of 48 weaned castrated male pigs (Duroc $\times$ Large White $\times$ Landrace, $8.12 \pm 0.56$ $\mathrm{kg}$, weaned at $21 \pm 2 \mathrm{~d}$ of age ) were purchased from the Hubei Zhengda Swine Co., Ltd (Wuhan, Hubei, China), and individually housed in the stainless steel cages (length $1.08 \mathrm{~m}$, width $0.98 \mathrm{~m}$, height $0.84 \mathrm{~m}$ ). All pigs were housed in an environmentally controlled room (temperature $28^{\circ} \mathrm{C}$ and natural light), and allowed ad libitum access to water and feed during a 19-d experimental period.

\section{Experimental design}

Based on the BW, forty-eight piglets were randomly assigned to four treatments (two sampling times), i) non-challenged control (CTRL; piglets received a control diet and injected with $0.9 \% \mathrm{NaCl}$ solution); ii) lipopolysaccharide (LPS)-challenged control (LPSCC; piglets received the same control diet and injected with Escherichia coli LPS ); iii) LPS+0.5\% Asn treatment (piglets received a $0.5 \%$ Asn diet and injected with LPS); and iv) LPS+1.0\% Asn treatment (piglets received a 1.0\% Asn diet and injected with LPS). There were six replicates (n =6) for each treatment in each sampling time, and each replicate had one pig. In this study, the dose of the Asn (L-Asn, purity >99\%; Amino Acid Bio-Chemical Co, Wuhan, China) and LPS (Escherichia coli serotype 055: B5, Sigma Chemical Inc., St. Louis, MO, USA) were determined on the basis of our previous studies [14]. The control diet was prepared to meet or exceed NRC [15] nutrient requirements (Table 1), and the diets were isonitrogenous by supplementation of L-
Table 1. Ingredients and nutritional composition of diet (as fed basis)

\begin{tabular}{|c|c|}
\hline Items & Content \\
\hline \multicolumn{2}{|l|}{ Ingredients (\%) } \\
\hline Corn & 55.50 \\
\hline Soybean meal (44\% crude protein) & 22.00 \\
\hline Wheat bran & 3.00 \\
\hline Fish meal & 5.50 \\
\hline Corn oil or fish oil & 5.00 \\
\hline Soy protein concentrate & 2.50 \\
\hline Milk-replacer powder & 3.00 \\
\hline Limestone & 0.70 \\
\hline Dicalcium phosphate & 1.00 \\
\hline Salt & 0.20 \\
\hline L-Lysine.HCI (78.8\% Lysine) & 0.27 \\
\hline Acidifier ${ }^{1)}$ & 0.20 \\
\hline Butylated hydroquinone & 0.05 \\
\hline Preservative ${ }^{2)}$ & 0.05 \\
\hline Sweetener ${ }^{3)}$ & 0.03 \\
\hline Vitamin and mineral premix ${ }^{4)}$ & 1.00 \\
\hline \multicolumn{2}{|l|}{ Nutrient composition (g/kg) } \\
\hline Digestible energy) (MJ/kg) & 14.0 \\
\hline Crude protein $^{6}$ & 202 \\
\hline (alcium ${ }^{6)}$ & 9.0 \\
\hline Phosphorus ${ }^{6)}$ & 7.0 \\
\hline Lysine $^{6)}$ & 13.5 \\
\hline Methionine+cysteine ${ }^{6)}$ & 7.2 \\
\hline Aspartate+asparagine $^{6)}$ & 16.9 \\
\hline \multicolumn{2}{|c|}{ 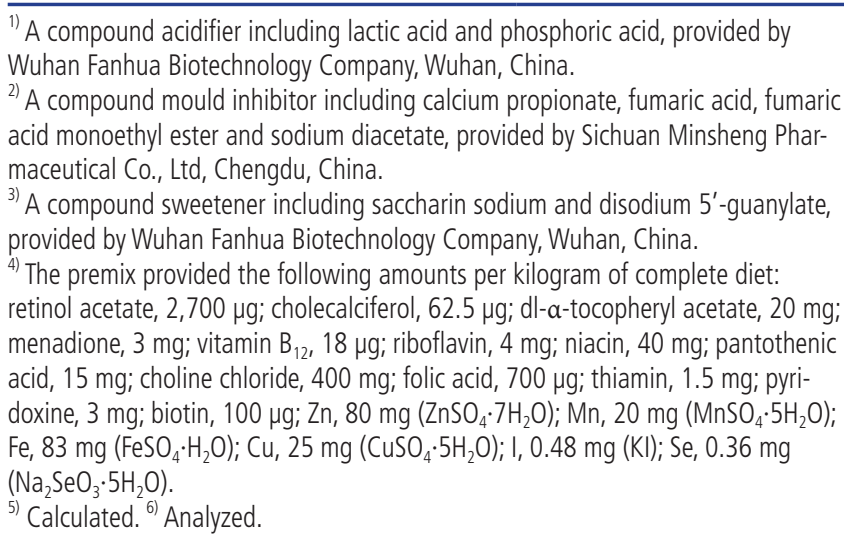 } \\
\hline
\end{tabular}

alanine (purity >99\%; Amino Acid Bio-Chemical Co, China). During the entire $19 \mathrm{~d}$ feeding trial (pre-challenge), there were no differences in average daily gain, average daily feed intake and feed:gain ratio among the four treatment groups (data not shown). On d 19, the LPS-challenged pigs were injected intraperitoneally with $500 \mu \mathrm{g} / \mathrm{mL}$ Escherichia coli LPS at 100 $\mu \mathrm{g} / \mathrm{kg} \mathrm{BW}(0.2 \mathrm{~mL} / \mathrm{kg} \mathrm{BW})$, and the pigs in control group were injected with the same volume of $0.9 \% \mathrm{NaCl}$ solution. LPS injection caused fever, somnolence, anorexia, vomiting, and shivering within $1 \mathrm{~h}$ in all pigs. At $4 \mathrm{~h}$ or $24 \mathrm{~h}(\mathrm{n}=6)$ after LPS or saline injection, pigs were slaughtered under anesthesia with an intravenous injection of sodium pentobarbital $(50 \mathrm{mg} / \mathrm{kg}$ $\mathrm{BW}$ ), and a portion of the liver (approximately $3 \mathrm{~g}$ ) was re- 
moved and frozen in liquid nitrogen immediately. One part of the liver sample (approximately $1 \mathrm{~g}$ ) was used for measuring ATP, ADP, and AMP concentrations, and another part (approximately $2 \mathrm{~g}$ ) was then stored at $-80^{\circ} \mathrm{C}$ for mRNA and protein expression analysis.

\section{ATP, ADP, and AMP concentrations in the liver}

The ATP, ADP, and AMP concentrations in the liver were measured with high performance liquid chromatography (HPLC) according to a published method [16]. Briefly, the supernatant was prepared from the liver. As for the HPLC, the detection wavelength was $260 \mathrm{~nm}$, and the column temperature and the pump flow was $35^{\circ} \mathrm{C}$ and $1.0 \mathrm{~mL} / \mathrm{min}$, respectively. Total adenine nucleotide (TAN) and adenylate energy charges (AEC) were calculated using the following equations: TAN $=\mathrm{ATP}+$ $\mathrm{ADP}+\mathrm{AMP} ; \mathrm{AEC}=(\mathrm{ATP}+0.5 \mathrm{ADP}) /(\mathrm{ATP}+\mathrm{ADP}+\mathrm{AMP})[16]$.

\section{mRNA abundance analysis by real-time PCR}

Total RNA was isolated from pig liver by Trizol (\#9108, TaKaRa Biotechnology [Dalian] Co., Ltd., Dalian, China). cDNA was synthesized with Prime Script RT reagent kit with gDNA eraser according to the producer instructions (\#RR047A, TaKaRa Biotechnology [Dalian] Co., Ltd., China). The expression of the target genes were analyzed by the real-time polymerase chain reaction (PCR) with an Applied Biosystems 7500 RealTime PCR System (Applied Biosystems, Life Technologies, Foster City, CA, USA) using a SYBR Premix Ex TaqTM (Tli RNaseH Plus) qPCR kit (\#RR420A, TaKaRa Biotechnology [Dalian] Co., Ltd., China). The PCR program was as follows: $95^{\circ} \mathrm{C}$ for $30 \mathrm{~s}$, followed by 40 cycles of $95^{\circ} \mathrm{C}$ for $5 \mathrm{~s}$ and $60^{\circ} \mathrm{C}$ for $34 \mathrm{~s}$. The target genes were amplified by using the forward and reverse primers, which were designed with Primer Premier 6.0 and synthesized by TaKaRa Biotechnology (China,
Table 2), and their mRNA expression relative to housekeeping gene (glyceraldehyde-3-phosphate dehydrogenase) were calculated according to the $2^{-\Delta \Delta \mathrm{CT}}$ method [17].

\section{Protein abundance analysis by Western blot}

The method for quantification of liver protein expression was carried out with Western blot as previously described [13]. Briefly, equal amounts of proteins in liver supernatant fluids were separated on $12 \%$ sodium dodecyl sulfate-polyacrylamide gel electrophoresis and then transferred to blotting membrane for immunoblotting. The specific primary antibodies included rabbit phosphorylated AMPKa (pAMPKa; Thr172; 1:1,000, \#2532) and rabbit anti-total AMPKa (tAMPKa; 1:1,000,\#2535) (Cell Signaling Technology Inc., Danvers, MA, USA).

\section{Statistical analysis}

The experimental data were analyzed using SPSS 17.0 software (SPSS Inc., Chicago, IL, USA). T test was used to determine whether there was a significant difference between the data obtained in the LPS group (LPSCC, $0 \%$ Asn) and the data obtained in the control group, in order to determine the effect of LPS challenge on the weaned piglets. Linear and quadratic polynomial contrasts were used to determine the response to Asn supplementation among LPS-challenged pigs. All data were expressed as means \pm SE. The statistical significance level for all analyses was set at $\mathrm{p} \leq 0.05$ and $0.05<\mathrm{p}<0.10$ were considered as trends.

\section{RESULTS}

Concentrations of ATP, ADP, and AMP in the liver As shown in Table 3, LPS challenge had no effect on the ATP, ADP, and AMP concentrations ( $p>0.1$ ) at 4 or 24 h post-in-

Table 2. Specific primer sequences for pigs used for real-time polymerase chain reaction

\begin{tabular}{|c|c|c|c|c|}
\hline Gene & Forward $\left(5^{\prime}-3^{\prime}\right)$ & Reverse $\left(5^{\prime}-3^{\prime}\right)$ & Gene Bank No. & Sequences bp \\
\hline Hexok 2 & CTCATCACAACCGTTACCA & TGTCATTAGTGTCCTCATCC & NM_001122987.1 & 119 \\
\hline L-PFK & CTGCACCGCATCATGGA & CCCCATCACCTCCAGAACA & XM_003358990.1 & 84 \\
\hline$P K$ & TCACTCCACAGACCTCAT & TACCTAGCCACCTGATGT & XM_003356683.1 & 123 \\
\hline PDH & GCAGACTTACCGTTACCAT & GATAGCCGAGTTCTTCCAA & XM_003360244.2 & 248 \\
\hline$A C O$ & CTCGCAGACCCAGATGAAAT & TCCAAGCCTCGAAGATGAGT & AF185048 & 218 \\
\hline L-CPT-1 & GGACCGCCACCTGTTCTGCCTCTA & GCCCCCTCCGCTCGACACATAC & AF288789 & 175 \\
\hline$C S$ & TCTCAGCTCAGTGCAGCCATTACA & CTGCAACACAAGGTAGCTTTGCGA & NM_214276 & 145 \\
\hline$I C D H \beta$ & TGTGGTTCCTGGTGAGAG & CGAGATTGAGATGCCGTAG & XM_003361597.1 & 149 \\
\hline$I C D H \gamma$ & GGTGGAGAGCCTCAAGAT & TGGTGGTGTTGTCTACGA & XM_003360495.1 & 218 \\
\hline AMPKa 1 & AAATCGGCCACTACATCCTG & GGATGCCTGAAAAGCTTGAG & NM_001167633.1 & 187 \\
\hline AMPKa2 & AACATGGACGGGTTGAAGAG & CGCAGAAACTCACCATCTGA & NM_214266.1 & 193 \\
\hline Sirt1 & CTGGAACAGGTTGCAGGAAT & CCTAGGACATCGAGGAACCA & EU030283 & 144 \\
\hline$P G C 1 a$ & GATGTGTCGCCTTCTTGTTC & CATCCTTTGGGGTCTTTGAG & NM_213963 & 93 \\
\hline GAPDH & CGTCCCTGAGACACGATGGT & GCCTTGACTGTGCCGTGGAAT & AF017079 & 194 \\
\hline
\end{tabular}

Hexok 2, hexokinase 2; L-PFK, 6-phosphofructokinase (liver type-like); $P K$, pyruvate kinase; $P D H$, pyruvate dehydrogenase; $A C O$, acyl-coenzyme A oxidase; L-CPT-1, liver carnitine palmitoyltransferase I; CS, Citrate synthase; ICDH $\beta$, isocitrate dehydrogenase $\beta ; / C D H ~ \gamma$, isocitrate dehydrogenase $\gamma_{i}$ AMPK, AMP-activated protein kinase; Sirt1, silent information regulator 1; $P G C 1 \alpha$, peroxisome proliferator activated receptor gamma coactivator-1a; GAPDH, glyceraldehyde-3-phosphate dehydrogenase. 
Table 3. The effect of Asn supplementation on the ATP production in liver at $24 \mathrm{~h}$ after Escherichia coli lipopolysaccharide challenge in weaned pigs ${ }^{1)}$

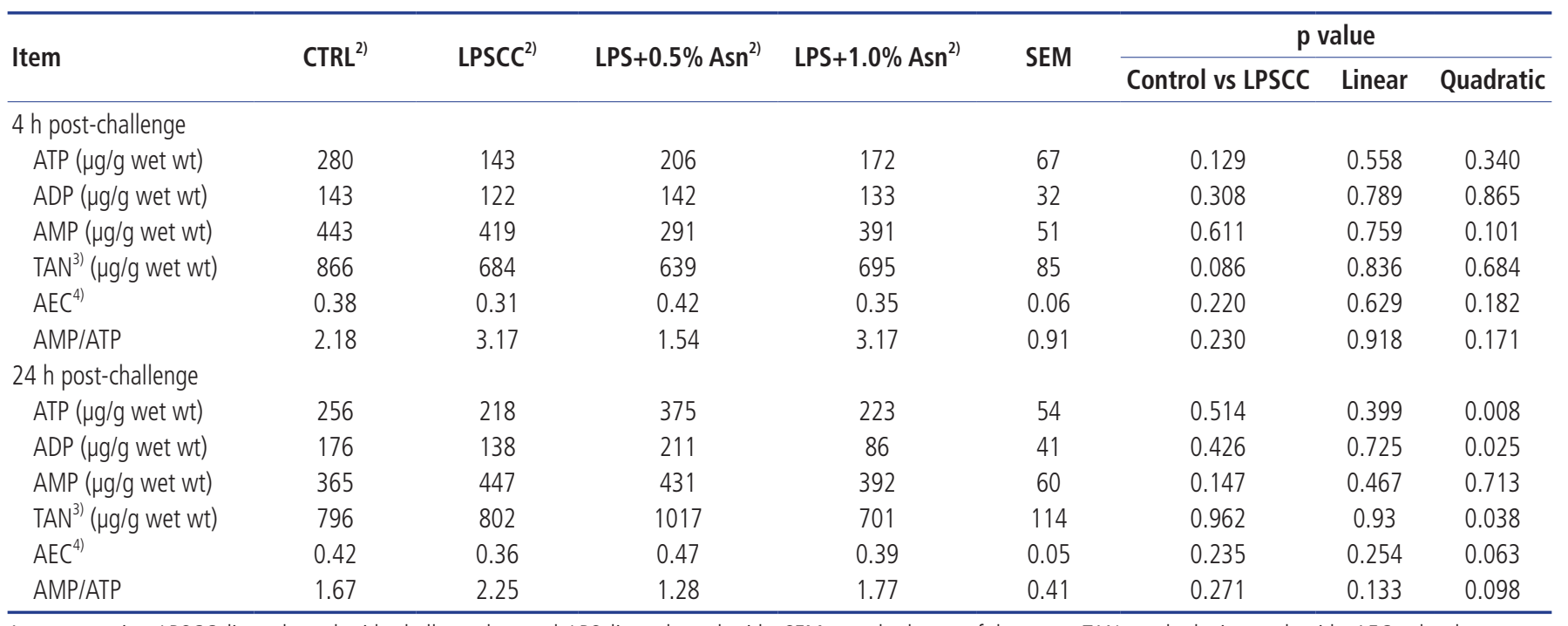

Asn, asparagine; LPSCC, lipopolysaccharide challenged control; LPS, lipopolysaccharide; SEM, standard error of the mean; TAN, total adenine nucleotide; AEC, adenylate energy charges.

1) Values are means $\pm S E, n=6$ (1 pig/pen).

${ }^{2)}$ CTRL (non-challenged control), piglets receiving a control diet and injected with $0.9 \% \mathrm{NaCl}$ solution; LPSCC (LPS challenged control), piglets receiving the same control diet and injected with Escherichia coli LPS; LPS+0.5\% Asn, piglets receiving a 0.5\% Asn diet and injected with LPS; LPS $+1.0 \%$ Asn, piglets receiving a 1.0\% Asn diet and injected with LPS.

3) $T A N=A T P+A D P+A M P$.

4) $A E C=(A T P+0.5 A D P) /(A T P+A D P+A M P)$.

jection. Dietary supplementation with Asn had no effect on the concentrations of ATP, ADP, and AMP at $4 \mathrm{~h}$ after LPS challenge ( $p>0.1)$, however, dietary Asn supplementation increased ATP and ADP concentrations (quadratic, $\mathrm{p}<0.05$ ), and had a tendency to increase AEC (quadratic, $p<0.1$ ) and reduce AMP/ATP ratio (quadratic, $\mathrm{p}<0.1$ ) at $24 \mathrm{~h}$ after LPS challenge.

\section{mRNA expression of genes related to the energy metabolism}

As shown in Table 4, at $4 \mathrm{~h}$ post-injection, LPS challenge upregulated the mRNA expression of liver hexokinase (Hexok) 2 , and down-regulated the mRNA expression of 6-phosphofructokinase (liver type-like) (L-PFK), pyruvate dehydrogenase (PDH), acyl-coenzyme A oxidase, and liver carnitine palmitoyltransferase (L-CPT)-1 ( $\mathrm{p}<0.05)$. Asn supplementation had no effect on mRNA expression of the key enzymes involved in glycolysis, TCA cycle and fatty acid oxidation after $4 \mathrm{~h}$ LPS challenge ( $p>0.1)$. However, at $24 \mathrm{~h}$ post-challenge, LPS challenge decreased mRNA expressions of PDH and citrate synthase (CS) $(\mathrm{p}<0.05)$. Asn supplementation increased pyruvate kinase (PK), $\mathrm{PDH}, \mathrm{CS}$, and isocitrate dehydrogenase (ICDH) $\beta \mathrm{mRNA}$ expression (linear, $\mathrm{p}<0.05$; quadratic, $\mathrm{p}<0.05$ ), and had a tendency to increase Hexok 2 mRNA expression (linear, $0.05<\mathrm{p}$ $<0.1)$ at $24 \mathrm{~h}$ after LPS challenge.

mRNA expression of AMPKa1, AMPKa2, Sirt1, and PGC1a
As shown in Table 5, LPS challenge decreased the mRNA expression of AMP-activated protein kinase (AMPK) a1, AMPKa2, and silent information regulator (Sirt) 1 at $24 \mathrm{~h}$ post-LPS challenge. However, Asn supplementation had no effect on the mRNA expression of AMPKa1, AMPKa2, Sirt1, and PGC1 $\alpha$ at $4 \mathrm{~h}$ or $24 \mathrm{~h}$ after LPS challenge.

\section{Protein phosphorylation and abundance of AMPKa}

As shown in Table 6, LPS challenge had no effect on the pAMPKa/tAMPKa ratio $(\mathrm{p}>0.05)$ at 4 and $24 \mathrm{~h}$ post-injection. However, Asn supplementation increased this ratio at $24 \mathrm{~h}$ after LPS challenge (linear, $\mathrm{p}<0.05$; quadratic, $\mathrm{p}<0.05$ ).

\section{DISCUSSION}

Many amino acids can be used as an energy source by the body, such as glutamine [18], proline [19,20], arginine [21], and aspartate $[13,22]$. Yi et al [23] found dietary $\mathrm{N}$-acetylcysteine supplementation also could alleviate liver injury in lipopolysaccharide-challenged piglets by improving energy metabolism.

ATP is the main energy source in a variety of cellular functions. Ramesh et al [24] reported that Asn increased the ODC activity, which primarily controls the intracellular concentrations of polyamines. Fausto [25] also found that Asn could increase ODC activity in regenerating liver. Polyamines play an essential role in regulating energy metabolism [26]. In our study, Asn supplementation increased ATP concentration at 
Table 4. The effect of Asn supplementation on the liver carbohydrate metabolism and tricarboxylic acid cycle at $24 \mathrm{~h}$ after Escherichia coli LPS challenge in weaned pigs ${ }^{1)}$

\begin{tabular}{|c|c|c|c|c|c|c|c|c|}
\hline \multirow{2}{*}{ Item } & \multirow{2}{*}{$\mathrm{CTRL}^{2)}$} & \multirow{2}{*}{ LPSCC $^{2)}$} & \multirow{2}{*}{ LPS+0.5\% Asn ${ }^{2)}$} & \multirow{2}{*}{ LPS+1.0\% Asn ${ }^{2)}$} & \multirow{2}{*}{ SEM } & \multicolumn{3}{|c|}{$p$ value } \\
\hline & & & & & & Control vs LPSCC & Linear & Quadratic \\
\hline \multicolumn{9}{|c|}{$4 \mathrm{~h}$ post-challenge } \\
\hline \multicolumn{9}{|c|}{ Carbohydrate metabolism } \\
\hline Hexok 2 & 1.00 & 15.9 & 21.3 & 25.57 & 5.13 & 0.001 & 0.120 & 0.293 \\
\hline L-PFK & 1.00 & 0.44 & 0.69 & 0.52 & 0.14 & 0.002 & 0.291 & 0.163 \\
\hline PK & 1.00 & 1.67 & 1.46 & 1.54 & 0.27 & 0.399 & 0.573 & 0.787 \\
\hline $\mathrm{PDH}$ & 1.00 & 0.90 & 0.80 & 0.81 & 0.11 & 0.032 & 0.305 & 0.571 \\
\hline \multicolumn{9}{|c|}{ Fatty acid oxidation } \\
\hline $\mathrm{ACO}$ & 1.00 & 0.45 & 0.60 & 0.37 & 0.12 & 0.001 & 0.888 & 0.112 \\
\hline L-CPT-1 & 1.00 & 0.35 & 0.42 & 0.22 & 0.14 & 0.003 & 0.483 & 0.158 \\
\hline \multicolumn{9}{|c|}{ Tricarboxylic acid cycle } \\
\hline CS & 1.00 & 0.98 & 0.89 & 0.84 & 0.10 & 0.806 & 0.189 & 0.428 \\
\hline $\mathrm{ICDH} \beta$ & 1.00 & 0.90 & 0.94 & 0.77 & 0.12 & 0.397 & 0.460 & 0.348 \\
\hline $\mathrm{ICDH} \gamma$ & 1.00 & 0.83 & 0.79 & 0.77 & 0.10 & 0.194 & 0.511 & 0.811 \\
\hline \multicolumn{9}{|c|}{ 24h post-challenge } \\
\hline \multicolumn{9}{|c|}{ Carbohydrate metabolism } \\
\hline Hexok 2 & 1.00 & 0.59 & 1.30 & 1.15 & 0.33 & 0.095 & 0.069 & 0.134 \\
\hline L-PFK & 1.00 & 0.64 & 0.59 & 0.60 & 0.17 & 0.169 & 0.519 & 0.771 \\
\hline PK & 1.00 & 0.90 & 1.25 & 1.14 & 0.11 & 0.486 & 0.010 & 0.007 \\
\hline PDH & 1.00 & 0.67 & 0.81 & 0.95 & 0.10 & 0.027 & $<0.001$ & 0.001 \\
\hline \multicolumn{9}{|c|}{ Fatty acid oxidation } \\
\hline $\mathrm{ACO}$ & 1.00 & 0.69 & 0.65 & 0.70 & 0.18 & 0.258 & 0.868 & 0.741 \\
\hline L-CPT-1 & 1.00 & 0.32 & 0.16 & 0.24 & 0.30 & 0.163 & 0.211 & 0.194 \\
\hline \multicolumn{9}{|c|}{ Tricarboxylic acid cycle } \\
\hline CS & 1.00 & 0.68 & 0.99 & 0.95 & 0.11 & 0.039 & 0.014 & 0.033 \\
\hline $\mathrm{ICDH} \beta$ & 1.00 & 0.66 & 0.72 & 0.91 & 0.15 & 0.145 & 0.008 & 0.004 \\
\hline $\mathrm{ICDH} \gamma$ & 1.00 & 0.72 & 0.71 & 0.67 & 0.11 & 0.078 & 0.570 & 0.760 \\
\hline
\end{tabular}

Asn, asparagine; LPS, lipopolysaccharide; LPSCC, lipopolysaccharide challenged control; SEM, standard error of the mean; Hexok 2, hexokinase 2; L-PFK, 6-phosphofructokinase (liver type-like); PK, pyruvate kinase; PDH, pyruvate dehydrogenase; ACO, acyl-coenzyme A oxidase; L-CPT-1, liver carnitine palmitoyltransferase I; CS, Citrate synthase; ICDH $\beta$, isocitrate dehydrogenase $\beta ;$ ICDH $\gamma$, isocitrate dehydrogenase $\gamma$.

1) Values are means, $n=6$ ( 1 pig/pen).

${ }^{2)}$ CTRL (non-challenged control), piglets receiving a control diet and injected with $0.9 \% \mathrm{NaCl}$ solution; LPSCC (LPS challenged control), piglets receiving the same control diet and injected with Escherichia coli LPS; LPS+0.5\% Asn, piglets receiving a 0.5\% Asn diet and injected with LPS; LPS+1.0\% Asn, piglets receiving a 1.0\% Asn diet and injected with LPS.

Table 5. The effect of Asn supplementation on the liver mRNA expression of AMPKa1, AMPKa2, Sirt1, and PGC1 $a$ at $4 \mathrm{~h}$ or $24 \mathrm{~h}$ after LPS challenge in weaned piglets ${ }^{1)}$

\begin{tabular}{|c|c|c|c|c|c|c|c|c|}
\hline \multirow{2}{*}{ Item } & \multirow{2}{*}{ CTRL $^{2)}$} & \multirow{2}{*}{ LPSCC $^{2)}$} & \multirow{2}{*}{$\mathrm{LPS}+0.5 \% \mathrm{Asn}^{2)}$} & \multirow{2}{*}{ LPS+1.0\% Asn ${ }^{2)}$} & \multirow{2}{*}{ SEM } & \multicolumn{3}{|c|}{$p$ value } \\
\hline & & & & & & Control vs LPSCC & Linear & Quadratic \\
\hline \multicolumn{9}{|c|}{$4 \mathrm{~h}$ post-challenge } \\
\hline AMPKa1 & 1.00 & 0.94 & 1.02 & 0.82 & 0.14 & 0.627 & 0.623 & 0.355 \\
\hline AMPKa2 & 1.00 & 0.99 & 1.01 & 0.89 & 0.19 & 0.945 & 0.715 & 0.798 \\
\hline Sirt1 & 1.00 & 1.17 & 1.06 & 0.91 & 0.24 & 0.495 & 0.326 & 0.580 \\
\hline $\mathrm{PGC1} 1 \alpha$ & 1.00 & 0.36 & 0.39 & 0.14 & 0.22 & 0.073 & 0.189 & 0.094 \\
\hline \multicolumn{9}{|c|}{$24 \mathrm{~h}$ post-challenge } \\
\hline AMPKa1 & 1.00 & 0.54 & 0.64 & 0.64 & 0.16 & 0.053 & 0.281 & 0.542 \\
\hline AMPKa2 & 1.00 & 0.47 & 0.44 & 0.53 & 0.17 & 0.052 & 0.723 & 0.659 \\
\hline Sirt1 & 1.00 & 0.61 & 0.46 & 0.60 & 0.13 & 0.039 & 0.608 & 0.277 \\
\hline $\mathrm{PGC1} 1 \alpha$ & 1.00 & 0.98 & 0.72 & 0.91 & 0.26 & 0.930 & 0.612 & 0.634 \\
\hline
\end{tabular}

Asn, asparagine; AMPK, AMP-activated protein kinase; Sirt1, silent information regulator 1; PGC1 $\alpha$, peroxisome proliferator activated receptor gamma coactivator-1 $\alpha$; LPS, lipopolysaccharide; LPSCC, lipopolysaccharide challenged control; SEM, standard error of the mean.

1) Values are means, $n=6$ (1 pig/pen).

${ }^{2)}$ CTRL (non-challenged control), piglets receiving a control diet and injected with $0.9 \% \mathrm{NaCl}$ solution; LPSCC (LPS challenged control), piglets receiving the same control diet and injected with Escherichia coli LPS; LPS+0.5\% Asn, piglets receiving a 0.5\% Asn diet and injected with LPS; LPS+1.0\% Asn, piglets receiving a 1.0\% Asn diet and injected with LPS. 
Table 6. The effect of Asn supplementation on the liver pAMPKa/tAMPKa ratio (AU) at $24 \mathrm{~h}$ after Escherichia coli LPS challenge in weaned piglets ${ }^{1)}$

\begin{tabular}{|c|c|c|c|c|c|c|c|c|}
\hline \multirow{2}{*}{ Item } & \multirow{2}{*}{$\mathrm{CTRL}^{2)}$} & \multirow{2}{*}{ LPSCC $^{2)}$} & \multirow{2}{*}{ LPS+0.5\% Asn ${ }^{2)}$} & \multirow{2}{*}{ LPS+1.0\% Asn ${ }^{2)}$} & \multirow{2}{*}{ SEM } & \multicolumn{3}{|c|}{$p$ value } \\
\hline & & & & & & Control vs LPSCC & Linear & Quadratic \\
\hline $4 \mathrm{~h}$ post-challenge & 16.79 & 16.11 & 19.02 & 20.90 & 5.22 & 0.897 & 0.326 & 0.586 \\
\hline $24 \mathrm{~h}$ post-challenge & 23.27 & 25.91 & 25.13 & 44.49 & 7.92 & 0.692 & 0.044 & 0.050 \\
\hline
\end{tabular}

Asn, asparagine; AMPK, AMP-activated protein kinase; LPS, lipopolysaccharide; LPSCC, lipopolysaccharide challenged control; SEM, standard error of the mean.

1) Values are means, $n=6$ (1 pig/pen).

${ }^{2)}$ CTRL (non-challenged control), piglets receiving a control diet and injected with $0.9 \% \mathrm{NaCl}$ solution; LPSCC (LPS challenged control), piglets receiving the same control diet and injected with Escherichia coli LPS; LPS $+0.5 \%$ Asn, piglets receiving a 0.5\% Asn diet and injected with LPS; LPS+1.0\% Asn, piglets receiving a 1.0\% Asn diet and injected with LPS.

$24 \mathrm{~h}$ after LPS challenge, which might have resulted from the increased polyamines concentration. Moreover, the rise of ATP level indicated that Asn could play an important role on recovery of injured liver.

The ATP generation in the liver mainly depends on the respiratory chain and glycolysis $[27,28]$, which could meet the energy requirement during the early response to a stress. The Hexok catalyzes glucose phosphorylation, which is the first step of glycolysis. In the present study, we found that Asn supplementation had a tendency to increase Hexok 2 mRNA expression after $24 \mathrm{~h}$ LPS challenge, which could stimulate the subsequent glycolysis steps to produce more ATP to improve the liver's response to stress after LPS challenge. Six-phosphofructokinase (PFK) is one of the key enzymes of glycolysis. ATP and AMP can down-regulate and up-regulate the activity of PFK, respectively [29]. However, Reinhart and Lardy [30] reported that this enzyme in liver could be inactive because of the lower physiological concentrations of ATP and AMP. This result is consistent with our findings that Asn supplementation could increase ATP level after LPS challenge, however, the increased ATP level could not be enough to stimulate liver PFK expression.

The PK is also a key regulatory enzyme in glycolysis. In erythrocytes, PK insufficiency can decrease ATP level, which might shorten their life span [31]. Roy et al [32] reported that PK deficiency increased susceptibility to Salmonella typhimurium infection in mice. In this study, Asn supplementation increased the PK mRNA expression at $24 \mathrm{~h}$ after LPS challenge, which might in turn augment the ATP production and have a positive function to alleviate the liver stress after LPS challenge. The PDH is not only the key enzyme in the TCA cycle, but also the first component enzyme of pyruvate dehydrogenase complex. PDH catalyzes pyruvate to produce acetyl coenzyme A (acetyl-CoA). This reaction is a main link between glycolysis and TCA cycle. In this study, Asn increased the PDH mRNA expression at $24 \mathrm{~h}$ after LPS challenge. Theoretically, this increased mRNA expression might increase the production of acetyl-CoA, which can produce the energy through the TCA cycle. These results are also in accordance with the result that Asn supplementation increased the ATP production.
The CS is the first enzyme in TCA cycle. Zhang et al [33] reported that the increased Asn could stimulate the CS activity. In this study, Asn supplementation increased the CS mRNA expression at $24 \mathrm{~h}$ after LPS challenge, which might result from the high Asn circulation concentration. Accordingly, the increased CS mRNA expression could stimulate TCA cycle to produce more ATP to improve the injured liver. The ICDH are also the key enzymes in TCA cycle. There are three ICDH in liver, including one cytosolic and two mitochondrial ones. The latter, including ICDH $\beta$ and ICDH $\gamma$, are the key enzymes against oxidative damage in mitochondria. Schanbacher et al [34] reported that ICDH level increased in cattle's serum of hepatic injury model. However, in the present study, Asn supplementation had no effect on their mRNA expression.

Mammalian AMPK is a main regulator of energy control [35], which can be activated by the high concentration of the AMP and the reversible phosphorylation [36]. AMPK is a heterotrimer consisting seven subunits [37]. The major phosphorylated site is the Thr172 phosphorylation within the AMPK $a$-subunit. In response, the activated AMPK can stimulate catabolic pathways to produce more ATP in the face of energy stress. Our previous studies have shown that Asn supplementation could improve the injured intestine induced by LPS via the AMPK signaling pathway $[38,39]$. In this study, we found that $1.0 \%$ Asn supplementation had no effect on the AMPK mRNA expression at 4 and $24 \mathrm{~h}$ after LPS challenge, however, it increased liver pAMPKa/tAMPKa ratio at $24 \mathrm{~h}$, which was in response to the decreased AMP-to-ATP ratio, indicating that Asn could increase AMPK phosphorylation to produce more ATP to improve energy status in the injured liver induced by LPS challenge.

Asn can be deaminated to form aspartate (Asp) in an ATPdependent amidation reaction [8]. Our previous study had shown that Asp could decrease the expressions of hepatic proinflammatory mediators at $4 \mathrm{~h}$ after LPS challenge, which could not stimulate the positive effects of Asp on the injured liver induced by LPS. In contrast, Asp could attenuate this injury resulting from the increased expressions of hepatic pro-inflammatory mediators at $24 \mathrm{~h}$ after LPS challenge. These responses might be part of the reason that Asn improved the energy status to attenuate the injured liver just at $24 \mathrm{~h}$ after LPS challenge 
due to the increased expressions of hepatic pro-inflammatory mediators. Moreover, because our previous study found only one time-point $(4 \mathrm{~h})$ might not the most appropriate time point to measure all the intestinal pro-inflammatory mediators following the LPS challenge, in this study, we chose two time points for liver sample collection to analyze energy metabolism in liver based on our found in Asp [13].

\section{CONCLUSION}

In the present study, we showed that dietary supplementation with Asn had no effect on the energy status at $4 \mathrm{~h}$ after LPS injection. However, Asn could improve the energy status in injured liver at $24 \mathrm{~h}$ after LPS challenge, as evidenced by increased liver content of ATP, which might result from increasing the activity of TCA cycle and glycolysis, and Asn supplementation could stimulate AMPK phosphorylation. The results indicated that Asn could play an important role in increasing energy production just at the late stage of LPS challenge. Furthermore, in our study, it remained unclear whether the positive effects on energy metabolism were mediated directly by Asn itself or by its metabolites.

\section{CONFLICT OF INTEREST}

We certify that there is no conflict of interest with any financial organization regarding the material discussed in the manuscript.

\section{ACKNOWLEDGMENTS}

This study was financially supported by the National Natural Science Foundation of China (31422053 and 31372318).

\section{REFERENCES}

1. Crosby LO, Cline TR. Effect of asparagine on growth and protein synthesis in weanling rats. J Anim Sci 1973;37:713-7.

2. Rhoads JM, Argenzio RA, Chen W, Gomez GG. Asparagine stimulates piglet intestinal $\mathrm{Cl}$ - secretion by a mechanism requiring a submucosal glutamate receptor and nitric oxide. J Pharmacol Exp Ther 1995;274:404-12.

3. Kandil HM, Argenzio RA, Chen W, et al. L-glutamine and L-asparagine stimulate ODC activity and proliferation in a porcine jejunal enterocyte line. Am J Physiol 1995;269:G591-9.

4. Ray RM, Viar MJ, Patel TB, Johnson LR. Interaction of asparagine and EGF in the regulation of ornithine decarboxylase in IEC-6 cells. Am J Physiol 1999;276:G773-80.

5. Milman HA, Cooney DA, Huang CY. Studies on the mechanism of the glutamine-dependent reaction catalyzed by asparagine synthetase from mouse pancreas. J Biol Chem 1980;255: 1862-6.
6. Avramis VI, Panosyan EH. Pharmacokinetic/pharmacodynamic relationships of asparaginase formulations: the past, the present and recommendations for the future. Clin Pharmacokinet 2005;44:367-93.

7. Balasubramanian MN, Butterworth EA, Kilberg MS. Asparagine synthetase: regulation by cell stress and involvement in tumor biology. Am J Physiol Endocrinol Metab 2013;304:E78999.

8. Milman HA, Cooney DA. Partial purification and properties of L-asparagine synthetase from mouse pancreas. Biochem J 1979;181:51-9.

9. Viollet B, Foretz M, Guigas B, et al. Activation of AMP-activated protein kinase in the liver: a new strategy for the management of metabolic hepatic disorders. J Physiol 2006;574:41-53.

10. Masaki T, Chiba S, Tatsukawa H, et al. Adiponectin protects LPS-induced liver injury through modulation of TNF- $\alpha$ in KK-Ay obese mice. Hepatology 2004;40:177-84.

11. Alipour M, Omri A, Smith MG, Suntres ZE. Prophylactic effect of liposomal $\mathrm{N}$-acetylcysteine against LPS-induced liver injuries. J Endotoxin Res 2007;13:297-304.

12. Xu L, Scheenen WJ, Roubos EW, Kozicz T. Peptidergic EdingerWestphal neurons and the energy-dependent stress response. Gen Comp Endocrinol 2012;177: 296-304.

13. Kang P, Liu Y, Zhu H, et al. The effect of aspartate on the energy metabolism in the liver of weanling pigs challenged with lipopolysaccharide. Eur J Nutr 2015;54:581-8.

14. Li S, Liu YL, Shi HF, et al Effect of asparagine acid on growth performance, blood cell differential count, and blood biochemical measurements of weaned piglets after lipopolysaccharide challenge. Chinese J Anim Nutr 2012;24:2450-8.

15. NRC. Nutrient requirements of swine. 11th edition. Washington, DC, USA: National Academic Press; 2012.

16. Hou Y, Yao K, Wang L, et al. Effects of $\alpha$-ketoglutarate on energy status in the intestinal mucosa of weaned piglets chronically challenged with lipopolysaccharide. Br J Nutr 2011;106:357-63.

17. Livak KJ, Schmittgen TD. Analysis of relative gene expression data using real-time quantitative PCR and $2^{-\triangle \Delta C T}$ method. Methods 2001;25:402-8.

18. Zielke HR, Zielke CL, Ozand PT. Glutamine: a major energy source for cultured mammalian cells. Fed Proc 1984;43:121-5.

19. Scaraffia PY, Wells MA. Proline can be utilized as an energy substrate during flight of Aedes aegypti females. J Insect Physiol 2003;49:591-601.

20. Andrade VS, Rojas DB, de Andrade RB, et al. A possible antiinflammatory effect of proline in the brain cortex and cerebellum of rats. Mol Neurobiol 2017 Jun 5 [Epub]. https:/doi. org/10.1007/s12035-017-0626-Z

21. Hristina K, Langerholc T, Trapecar M. Novel metabolic roles of L-arginine in body energy metabolism and possible clinical applications. J Nutr Health Aging 2014;18:213-8.

22. Pi D, Liu Y, Shi H, et al. Dietary supplementation of aspartate enhances intestinal integrity and energy status in weanling 
piglets after lipopolysaccharide challenge. J Nutr Biochem 2014;25:456-62.

23. Yi D, Hou Y, Wang L, et al. Dietary N-acetylcysteine supplementation alleviates liver injury in lipopolysaccharide-challenged piglets. Br J Nutr 2014;111:46-54.

24. Ray RM, Viar MJ, Johnson LR. Amino acids regulate expression of antizyme-1 to modulate ornithine decarboxylase activity. J Biol Chem 2012;287:3674-90.

25. Fausto N. The control of ornithine decarboxylase activity during liver regeneration. Biochim Biophys Acta 1971;238:116-28.

26. Pirinen E, Kuulasmaa T, Pietilä M, et al. Enhanced polyamine catabolism alters homeostatic control of white adipose tissue mass, energy expenditure, and glucose metabolism. Mol Cell Biol 2007;27:4953-67.

27. Soboll S. Regulation of energy metabolism in liver. J Bioenerg Biomembr 1995;27:571-82.

28. Wang J, Li GR, Tan BE, et al. Oral administration of putrescine and proline during the suckling period improves epithelial restitution after early weaning in piglets. J Anim Sci 2015;93: 1679-88.

29. Furuya E, Uyeda K. An activation factor of liver phosphofructokinase. Proc Nati Acad Sci USA 1980;77:5861-4.

30. Reinhart GD, Lardy HA. Rat liver phosphofructokinase: kinetic activity under near-physiological conditions. Biochemistry 1980;19:1477-84.

31. Zanella A, Fermo E, Bianchi P, Valentini G. Red cell pyruvate kinase deficiency: molecular and clinical aspects. Br J Haematol 2005;130:11-25.

32. Roy MF, Riendeau N, Bédard C, et al. Pyruvate kinase defi ciency confers susceptibility to Salmonella typhimurium infection in mice. J Exp Med 2007;204:2949-61.

33. Zhang J, Fan J, Venneti S, et al. Asparagine plays a critical role in regulating cellular adaptation to glutamine depletion. Mol Cell 2014;56:205-18.

34. Schanbacher FL, Willett LB, Moorhead PD. Ornithine decarboxylase, serum isocitrate dehydrogenase and clinical chemisty changes during thioacetamide-induced hepatotoxicity in a calf. J Anim Sci 1981;53:1658-70.

35. Kahn BB, Alquier T, Carling D, Hardie DG. AMP-activated protein kinase: ancient energy gauge provides clues to modern understanding of metabolism. Cell. Metab 2005;1:15-25.

36. Stein SC, Woods A, Jones NA, Davison MD, Carling D. The regulation of AMP-activated protein kinase by phosphorylation. Biochem J 2000;345:437-43.

37. Merrill JF, Thomson DM, Hardman SE, et al. Iron deficiency causes a shift in AMP-activated protein kinase (AMPK) subunit composition in rat skeletal muscle. Nutr Metab (Lond) 2012;9: 104.

38. Wang XY, Liu YL, Li S, et al. Asparagine attenuates intestinal injury, improves energy status and inhibits AMP-activated protein kinase signalling pathways in weaned piglets challenged with Escherichia coli lipopolysaccharide. Br J Nutr 2015; 114:553-65.

39. Wu HT, Liu YL, Pi DA, et al. Asparagine attenuates hepatic injury caused by lipopolysaccharide in weaned piglets associated with modulation of Toll-like receptor 4 and nucleotidebinding oligomerisation domain protein signalling and their negative regulators. Br J Nutr 2015;114:189-201. 